\title{
UNIQUENESS OF GRIM HYPERPLANES FOR MEAN CURVATURE FLOWS
}

\author{
DITTER TASAYCO AND DETANG ZHOU
}

\begin{abstract}
In this paper we show that an immersed nontrivial translating soliton for mean curvature flow in $\mathbb{R}^{n+1}(n=2,3)$ is a grim hyperplane if and only if it is mean convex and has weighted total extrinsic curvature of at most quadratic growth. For an embedded translating soliton $\Sigma$ with nonnegative scalar curvature, we prove that if the mean curvature of $\Sigma$ does not change signs on each end, then $\Sigma$ must have positive scalar curvature unless it is either a hyperplane or a grim hyperplane.
\end{abstract}

\section{INTRODUCTION}

A mean curvature flow (MCF) in $\mathbb{R}^{n+1}$ is the negative gradient flow of the volume functional, which can be analyzed from the perspective of partial differential equations as shown by Huisken in [4]. MCF is smooth in a short time and singularities must happen over a longer time. According to the rate of blow-up of the second fundamental form $A(t, p)$ of the hypersurface $\Sigma_{t}$, this finite time singularity $T$ is called type-I, if there exists a constant $C_{0}$ such that

$$
\sup _{p \in \Sigma_{t}}|A(t, p)|^{2} \leq \frac{C_{0}}{(T-t)}
$$

for all $t<T$. Otherwise this finite time singularity is called type-II.

We will deal with translating solitons which are important in study of type-II singularities.

A complete connected isometrically immersed hypersurface $(\Sigma, \Phi)$ in $\mathbb{R}^{n+1}$ is called a translating soliton if its mean curvature vector satisfies

$$
\vec{H}=w^{\perp},
$$

where $w \in \mathbb{R}^{n+1}$ is a unitary vector and $w^{\perp}$ stands for the orthogonal projection of $w$ onto the normal bundle of $\Phi$. Let $\nu$ denote the unit normal along $\Phi$, then it is equivalent to

$$
H=-\langle\nu, w\rangle .
$$

In particular, considering $f: \mathbb{R}^{n+1} \longrightarrow \mathbb{R}$ defined by $f(x)=-\langle x, w\rangle$, then $\bar{\nabla} f=-w$ and $H=\langle\bar{\nabla} f, \nu\rangle$, therefore by definition translating solitons are $f$-minimal hypersurfaces. Since MCF is invariant under isometries,

2000 Mathematics Subject Classification. Primary: 53C21; Secondary: 53C42.

The second author was partially supported by CNPq and Faperj of Brazil. 
without loss of generality we may suppose that $w=(0, \ldots, 0,1)$, then the function $f$ is defined by $f(x)=-x_{n+1}$ and the $L_{f}$-stability operador of $\Sigma$ is given by

$$
L_{f}=\Delta_{f}+|A|^{2}
$$

There are some examples of translating solitons: vertical hyperplanes, grim hyperplanes, translating bowl solitons and translating catenoids. In this article we will give a characterization of grim hyperplanes in dimensions 2 and 3.

Recall that a grim hyperplane in $\mathbb{R}^{n+1}$ is a hypersurface $\mathcal{G}$ of $\mathbb{R}^{n+1}$ which can be represented parametrically via the embedding $\Phi:\left(-\frac{\pi}{2}, \frac{\pi}{2}\right) \times \mathbb{R}^{n-1} \longrightarrow$ $\mathbb{R}^{n+1}$ defined by

$$
\mathbf{\Phi}\left(t, y_{1}, \ldots, y_{n-1}\right)=\left(t, y_{1}, \ldots, y_{n-1},-\ln (\cos t)\right) .
$$

The grim hyperplane $\mathcal{G}$ satisfies the translating soliton equation with $w=$ $(0, \ldots, 0,1)$ i.e. it is $f$-minimal for $f\left(x_{1}, \ldots, x_{n+1}\right)=-x_{n+1}$. Also it has positive mean curvature. When $n=2$ or 3 , there exists a constant $C>0$ such that

$$
\int_{B_{R}}|A|^{2} e^{-f} \leq C R^{2}
$$

for all $R$ sufficiently large. The aim of this article is to prove that indeed the grim hyperplanes are the only ones with these properties when $n=2,3$.

Theorem 1. Let $\Phi: \Sigma^{n} \longrightarrow \mathbb{R}^{n+1}$ be a translating soliton, with $n=2$ or 3 , which is not a hyperplane. Then $\Sigma$ is a grim hyperplane if and only if $H=-\langle w, \nu\rangle \geq 0$ and there exists $C>0$ such that

$$
\int_{B_{R}}|A|^{2} e^{-f} \leq C R^{2}
$$

for all $R$ sufficiently large, where $B_{R}$ is the geodesic ball of radius $R$ and $f(x)=-\langle x, w\rangle$.

The expression (3) is not satisfied for $n \geq 4$ (see Proposition 11), thus Theorem 1 is sharp in this sense.

It has been known that if $H \geq 0$ on a translating soliton $\Sigma$, then either $H \equiv 0$ on $\Sigma$ and $\Sigma$ is a hyperplane, or $H>0$ everywhere on $\Sigma$. Note that both hyperplane and grim hyperplane has vanishing scalar curvature. In [6], Martín-Savas-Halilaj-Smoczyk proved that flat hyperplane and grim hyperplane are the only translating soliton with vanishing scalar curvature. It would be interesting to ask if the following is true.

Problem: Let $\Sigma$ be a translating soliton with nonnegative scalar curvature $S$. Is it true that either $S \equiv 0$ on $\Sigma$ and $\Sigma$ is a hyperplane or grim hyperplane, or $S>0$ everywhere on $\Sigma$ ?

This problem is related to a result proved by Huang-Wu in [3]. Let $M$ be a closed embedded $n$-dimensional hypersurface in $\mathbb{R}^{n+1}$ with nonnegative scalar curvature. Let $M_{t}$ be a solution to the mean curvature flow with 
initial hypersurface $M$. Then the scalar curvature of $M_{t}$ is strictly positive for all $t>0$.

For complete embedded translating solitons, we have

Theorem 2. Let $\left(\Sigma^{n}, g\right)$ be a embedded translating soliton with nonnegative scalar curvature $S$. Assume $H$ does not change signs on each end. Then either $\Sigma$ is a hyperplane or a grim hypersurface; or $\Sigma$ has positive scalar cuvature.

\section{Total Weighted EXtrinsic CURVAture}

In this section we will give the asymptotic properties of the total weighted extrinsic curvatures of grim hyperplanes. We have

$$
\partial_{t}=\sec (t)(\cos t, 0, \cdots, 0, \sin t) .
$$

We choose the unit normal $\nu$ to $\mathcal{G}$ to be $\nu=(\sin t, 0, \cdots, 0,-\cos t)$. A little computation shows that $\bar{\nabla}_{\partial_{t}} \nu=(\cos t) \partial_{t}$ and $\bar{\nabla}_{\partial_{y_{i}}} \nu=0 \quad(1 \leq i \leq n-1)$.

Then the principal curvatures are $\lambda_{1}=\cos t, \lambda_{2}=\ldots=\lambda_{n}=0$, thus on the coordinates $t, y_{1}, \ldots, y_{n-1}$ the mean curvature only depends on $t$ and is given by $H(t)=\cos t$. Since $t \in\left(-\frac{\pi}{2}, \frac{\pi}{2}\right)$, we have the norm of the second fundamental form is given by

$$
|A|(t)=\cos t=H(t) .
$$

Now, consider the function $f: \mathbb{R}^{n+1} \longrightarrow \mathbb{R}$ defined by $f(x)=-x_{n+1}$, then

$$
\langle\bar{\nabla} f, \nu\rangle=\cos t=H \text {. }
$$

Proposition 1. The Grim Hyperplane $\mathcal{G}$ in $\mathbb{R}^{n+1}$ satisfies

$$
\lim _{R \longrightarrow+\infty} \frac{1}{R^{n-1}} \int_{B_{R}}|A|^{2} e^{-f}=\left|B^{n-1}(1)\right| \pi,
$$

where $B_{R}$ is the geodesic ball with center at 0 and radius $R$ and $B^{n-1}$ (1) is the open ball in $\mathbb{R}^{n-1}$ of radius 1 and center at the origin.

Proof of Proposition 1. Observe that $f$ and the metric on $\mathcal{G}$ in the coordinates $t, y_{1}, \ldots, y_{n-1}$ are given by

$$
f(t)=\ln (\cos t)
$$

and

Thus

$$
g=\sec ^{2}(t) d t^{2}+d y_{1}^{2}+\ldots+d y_{n-1}^{2} .
$$

$$
r=\int_{0}^{t} \sec (\xi) d \xi=-\ln \left(\tan \left(\frac{1}{2}\left(\frac{\pi}{2}-t\right)\right)\right),
$$

we have $t=\frac{\pi}{2}-\eta(r)$, where $\eta(r)=2 \arctan \left(e^{-r}\right)$. Then

$$
g=d r^{2}+d y_{1}^{2}+\cdots+d y_{n-1}^{2} .
$$

Besides that $|A|$ and $f$ in the coordinates $r, y_{1}, \cdots, y_{n-1}$ are given by

$$
|A|(r)=\sin (\eta(r))
$$


and

$$
f(r)=\ln (\sin (\eta(r))) .
$$

Denoting by $\|$.$\| the standard norm of \mathbb{R}^{n-1}$, we have

$$
\begin{aligned}
B_{R} & =\left\{(r, y) \in \mathbb{R} \times \mathbb{R}^{n-1}: r^{2}+\|y\|^{2} \leq R^{2}\right\} \\
& =\left\{(r, y) \in \mathbb{R} \times \mathbb{R}^{n-1}:-\sqrt{R^{2}-\|y\|^{2}} \leq r \leq \sqrt{R^{2}-\|y\|^{2}}, \quad\|y\| \leq R\right\} .
\end{aligned}
$$

Since $-\eta^{\prime}(r)=\sin (\eta(r))$ is an even function, then

$$
\begin{aligned}
\int_{B_{R}}|A|^{2} e^{-f} & =\int_{\{\|y\| \leq R\}}\left[\int_{-\sqrt{R^{2}-\|y\|^{2}}}^{\sqrt{R^{2}-\|y\|^{2}}} \sin (\eta(r)) d r\right] d y \\
& =\int_{\{\|y\| \leq R\}}\left[\pi-2 \eta\left(\sqrt{R^{2}-\|y\|^{2}}\right)\right] d y \\
& =\pi \int_{\{\|y\| \leq R\}} 1 d y-2 \int_{\{\|y\| \leq R\}} \eta\left(\sqrt{R^{2}-\|y\|^{2}}\right) d y \\
& =\pi\left|B^{n-1}(1)\right| R^{n-1}-2 \int_{0}^{R}\left(\int_{\mathbb{S}_{\rho}^{n-2}} \eta\left(\sqrt{R^{2}-\rho^{2}}\right) d A\right) d \rho \\
& =\pi\left|B^{n-1}(1)\right| R^{n-1}-2 \operatorname{area}\left(\mathbb{S}^{n-2}\right) \int_{0}^{R} \eta\left(\sqrt{R^{2}-\rho^{2}}\right) \rho^{n-2} d \rho .
\end{aligned}
$$

where we have used the co-area formula. Now, letting $\rho=R \sin \theta$ and using the fact area $\left(\mathbb{S}^{n-2}\right)=(n-1)\left|B^{n-1}(1)\right|$, we have

$$
\frac{1}{R^{n-1}} \int_{B_{R}}|A|^{2} e^{-f}=\left|B^{n-1}(1)\right|\left[\pi-2(n-1) F_{n-1}(R)\right]
$$

where

$$
F_{n-1}(R)=\int_{0}^{\pi / 2} \eta(R \cos \theta) \sin ^{n-2} \theta \cos \theta d \theta .
$$

Observe that

$$
\lim _{R \longrightarrow+\infty} \eta(R \cos \theta) \sin ^{n-2} \theta \cos \theta=0 \quad \text { for all } \theta \in\left[0, \frac{\pi}{2}\right] .
$$

Fixing $R>0$, we have $\left|\eta(R \cos \theta) \sin ^{n-2} \theta \cos \theta\right| \leq \frac{\pi}{2} \sin ^{n-2} \theta \cos \theta$ for all $\theta \in[0, \pi / 2]$. Besides that

$$
\int_{0}^{\pi / 2} \sin ^{n-2} \theta \cos \theta d \theta=1 /(n-1) .
$$

Then $\lim _{R \longrightarrow+\infty} F_{n-1}(R)=0$, and hence by (5) , we get

$$
\lim _{R \longrightarrow+\infty} \frac{1}{R^{n-1}} \int_{B_{R}}|A|^{2} e^{-f}=\left|B^{n-1}(1)\right| \pi .
$$




\section{Proofs of Theorem 1 and Theorem 2}

We begin this section with the following lemma which is in a form more general than we need. The lemma may have its independent interest.

Lemma 1. Assume that on a complete weighted manifold $\left(M,\langle\rangle,, e^{-f} d \mathrm{voL}\right)$, the functions $u, v \in C^{2}(M)$, with $u>0$ and $v \geq 0$ on $M$, satisfy

$$
\Delta_{f} u+q(x) u \leq 0 \quad \text { and } \quad \Delta_{f} v+q(x) v \geq 0,
$$

where $q(x) \in C^{0}(M)$. Suppose that there exists a positive function $\kappa>0$ on $\mathbb{R}^{+}$satisfying $\frac{t}{\kappa(t)}$ is nonincreasing and

$$
\int^{+\infty} \frac{t}{\kappa(t)} d t=+\infty
$$

such that

$$
\int_{B_{R}} v^{2} e^{-f} \leq \kappa(R)
$$

for all $R$. Then there exists a constant $C$ such that $v=C u$.

Remark 1. Without loss of generality, we can assume $\kappa(t) \geq C\left(1+t^{2}\right)$. Some examples of $\kappa(t)$ are $C t^{2}, C t^{2} \log (1+t), C t^{2} \log (1+t) \log \log (3+t), \cdots$.

Proof of Lemma 1, Set $w=\frac{v}{u}$, then $v=w u$, thus by (7) we get

$$
\begin{aligned}
\Delta_{f} v & =w \Delta_{f} u+2\langle\nabla w, \nabla u\rangle+u \Delta_{f} w \\
& \leq-w(q u)+2\langle\nabla w, \nabla u\rangle+u \Delta_{f} w \\
& =-q v+2\langle\nabla w, \nabla u\rangle+u \Delta_{f} w .
\end{aligned}
$$

Then

$$
\Delta_{f} w \geq-2\langle\nabla w, \nabla(\ln u)\rangle .
$$

On the other hand, let $\varphi \in C_{o}^{2}(M)$, then by (9), we have

$$
\begin{aligned}
\int_{M} \varphi^{2}|\nabla w|^{2} e^{-f} & =\int_{M}\left\langle\varphi^{2} \nabla w, \nabla w\right\rangle e^{-f} \\
& =\int_{M}\left\langle\nabla\left(\varphi^{2} w\right), \nabla w\right\rangle e^{-f}-2 \int_{M} \varphi w\langle\nabla \varphi, \nabla w\rangle e^{-f} \\
& =-\int_{M} \varphi^{2} w\left(\Delta_{f} w\right) e^{-f}-2 \int_{M} \varphi w\langle\nabla \varphi, \nabla w\rangle e^{-f} \\
& \leq 2 \int_{M} \varphi^{2} w\langle\nabla w, \nabla(\ln u)\rangle e^{-f}-2 \int_{M} \varphi w\langle\nabla \varphi, \nabla w\rangle e^{-f} \\
& =2 \int_{M}\langle\varphi \nabla w, w(\varphi \nabla(\ln u)-\nabla \varphi)\rangle \\
& \leq \frac{1}{2} \int_{M} \varphi^{2}|\nabla w|^{2} e^{-f}+2 \int_{M} w^{2}|\varphi \nabla(\ln u)-\nabla \varphi|^{2} e^{-f}
\end{aligned}
$$


Then

$$
\int_{M} \varphi^{2}|\nabla w|^{2} e^{-f} \leq 4 \int_{M} w^{2}|\varphi \nabla(\ln u)-\nabla \varphi|^{2} e^{-f} \quad \forall \varphi \in C_{o}^{2}(M) .
$$

If $\psi \in C_{o}^{\infty}(M)$, then $\varphi=\psi u \in C_{o}^{2}(M)$. Besides that, a little computation shows

$$
\varphi \nabla(\ln u)-\nabla \varphi=-(\nabla \psi) u
$$

Thus, from (10), we have

$$
\begin{aligned}
\int_{M} \psi^{2} u^{2}|\nabla w|^{2} e^{-f} & \leq 4 \int_{M} w^{2}|\nabla \psi|^{2} u^{2} e^{-f} \\
& =4 \int_{M}|\nabla \psi|^{2} v^{2} e^{-f} \quad \forall \psi \in C_{o}^{\infty}(M) .
\end{aligned}
$$

Define functions $\beta, \xi$ on $[0,+\infty)$ as

$$
\beta(t):=\int_{0}^{t} \frac{\tau}{\kappa(\tau)} d \tau
$$

and $\xi$ is the inverse function of $\beta$. From the hypothesis we know $\beta^{\prime}$ is nonincreasing and $\xi^{\prime}$ is nondecreasing functions on $[0,+\infty)$. Now, we now choose a cutoff function

$$
\psi_{R}(x)= \begin{cases}1, & \text { on } B_{\xi(R)} ; \\ 2-\frac{\beta(r(x))}{R}, & \text { on } B_{\xi(2 R)} \backslash B_{\xi(R)} ; \\ 0, & \text { on } M \backslash B_{\xi(2 R) .}\end{cases}
$$

where $r(x)=d(x, p), p \in M$ is a fixed point and $B_{R}$ is the geodesic ball with radius $R$ and center $p$. We see that $\left|\nabla \psi_{R}\right|=\frac{\beta^{\prime}(r)}{R}=\frac{r}{R \kappa(r)}$. Then, by (8), we get

$$
\begin{aligned}
\int_{B_{\xi(R)}} u^{2}|\nabla w|^{2} e^{-f} & =\int_{B_{\xi(R)}} \psi_{R}^{2} u^{2}|\nabla w|^{2} e^{-f} \\
& \leq \int_{M} \psi_{R}^{2} u^{2}|\nabla w|^{2} e^{-f} \\
& \leq 4 \int_{M} v^{2}\left|\nabla \psi_{R}\right|^{2} e^{-f} \\
& =4 \int_{B_{\xi(2 R)} \backslash B_{\xi(R)}} v^{2}\left|\nabla \psi_{R}\right|^{2} e^{-f} \\
& =\frac{4}{R^{2}} \int_{\xi(R)}^{\xi(2 R)}\left(\beta^{\prime}(s)\right)^{2} \int_{\partial B_{s}} v^{2} e^{-f} d A d s
\end{aligned}
$$

Here we have used co-area formula. For convenience, we write $V(s)=$ $\int_{B_{s}} v^{2} e^{-f} d V$. Therefore

$$
V(s)=\int_{0}^{s} \int_{\partial B_{\tau}} v^{2} e^{-f} d A d \tau \leq \kappa(s)
$$


and

$$
\begin{aligned}
& \int_{B_{\xi(R)}} u^{2}|\nabla w|^{2} e^{-f} \leq \frac{4}{R^{2}} \int_{\xi(R)}^{\xi(2 R)}\left(\beta^{\prime}(s)\right)^{2} V^{\prime}(s) d s \\
& =\frac{4}{R^{2}}\left[V(s)\left(\beta^{\prime}(s)\right)^{2} \mid \begin{array}{l}
\xi(2 R) \\
\xi(R)
\end{array}-\int_{\xi(R)}^{\xi(2 R)} 2 V(s)\left(\beta^{\prime}(s)\right) d \beta^{\prime}(s)\right] \\
& \leq \frac{4}{R^{2}}\left[\begin{array}{l|l}
V(s)\left(\beta^{\prime}(s)\right)^{2} & \left.\begin{array}{l}
\xi(2 R) \\
\xi(R)
\end{array}-2 \int_{\xi(R)}^{\xi(2 R)} s d \beta^{\prime}(s)\right]
\end{array}\right.
\end{aligned}
$$

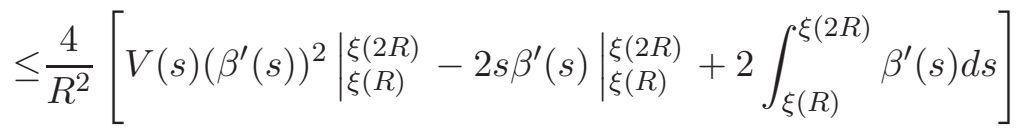

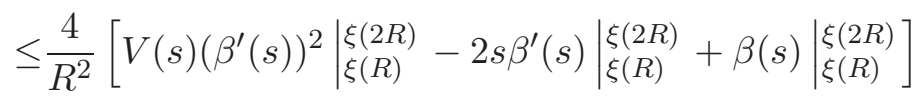

$$
\begin{aligned}
& \leq \frac{4}{R^{2}}\left[V(s)\left(\beta^{\prime}(s)\right)^{2}\left|\begin{array}{l|l}
\xi(2 R) \\
\xi(R)
\end{array}-2 s \beta^{\prime}(s)\right| \begin{array}{l}
\xi(2 R) \\
\xi(R)
\end{array}+R\right]
\end{aligned}
$$

Since

$$
V(s)\left(\beta^{\prime}(s)\right)^{2}=V(s) \beta^{\prime}(s) \beta^{\prime}(s) \leq s \beta^{\prime}(s),
$$

and $\beta^{\prime}(s)=\frac{s}{\kappa(s)}$, thus Remark 1 implies these terms are bounded, hence when $R \longrightarrow+\infty$, all the terms on the right hand side go to zero. So we get

$$
\int_{M} u^{2}|\nabla w|^{2} e^{-f}=0
$$

Then $\nabla w \equiv 0$, thus there is a constant $C$ such that $w \equiv C$ and hence $v=C u$.

Definition 1. A two-sided translating soliton $\Sigma$ is said to be stable if

$$
\int_{\Sigma}\left[|\nabla \varphi|^{2}-|A|^{2} \varphi^{2}\right] e^{-f} d \sigma \geq 0 \quad \text { for all } \varphi \in C_{o}^{\infty}(\Sigma) .
$$

As a consequence of Lemma 1, we have the following:

Corollary 1. Let $\Phi: \Sigma^{n} \longrightarrow \mathbb{R}^{n+1}$ be a stable translating soliton and let $\omega \in C^{2}(\Sigma)$ be a positive solution of the stability equation

$$
\Delta_{f} \omega+|A|^{2} \omega=0 .
$$

Moreover, if $H \geq 0$ and there exists a constant $C>0$ such that

$$
\int_{B_{R}} H^{2} e^{-f} \leq C R^{2} \quad \text { for all } R \text { large enough. }
$$

Then there exists a constant $\widetilde{C}$ such that $H=\widetilde{C} \omega$. In particular, if $H \neq \equiv 0$, then $\widetilde{C} \in \mathbb{R} \backslash\{0\}$ and $H>0$.

Now, we include here a result due to Li and Wang ([5]) which will be needed in the proof our main theorem. 
Lemma 2. Suppose $\Sigma$ is complete and there exists a nonnegative function $\varphi: \Sigma \longrightarrow \mathbb{R}$, not identically zero, such that $\left(\Delta_{f}+q\right)(\varphi) \leq 0$. Then $\Delta_{f}+q$ is stable.

Proof. Let $\Omega$ be a compact subdomain in $\Sigma$ and let $u$ be the first eigenfunction satisfying

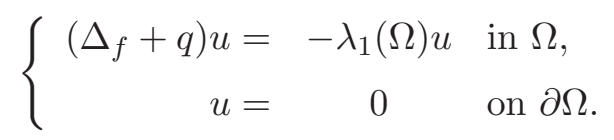

We may assume that $u \geq 0$ on $\Omega$. From regularity of $u$ and Hopf Lemma, we have

- $u>0$ in the interior of $\Omega$.

- $\frac{\partial u}{\partial \nu}<0$ on $\partial \Omega$, where $\nu$ is the outward unit normal of $\partial \Omega$.

Thus, integration by parts on $u$ and $\varphi$ and also the hypothesis, we have

$$
\begin{aligned}
\int_{\Omega} u\left(\Delta_{f} \varphi\right) e^{-f}-\int_{\Omega} \varphi\left(\Delta_{f} u\right) e^{-f} & =\int_{\partial \Omega} u \frac{\partial \varphi}{\partial \nu} e^{-f}-\int_{\partial \Omega} \varphi \frac{\partial u}{\partial \nu} e^{-f} \\
& =-\int_{\partial \Omega} \varphi \frac{\partial u}{\partial \nu} e^{-f} \geq 0
\end{aligned}
$$

From hypothesis and (14), we have

$$
\left\{\begin{aligned}
\Delta_{f} \varphi+Q \varphi & \leq 0 \\
\Delta_{f} u+Q u & =-\lambda_{1}(\Omega) u
\end{aligned}\right.
$$

Since $u>0$, multiplying the first inequality of (16) by $u$ and the second equation by $-\varphi$, and finally both by $e^{-f}$, we have

$$
u\left(\Delta_{f} \varphi\right) e^{-f}-\varphi\left(\Delta_{f} u\right) e^{-f} \leq \lambda_{1}(\Omega)(\varphi u) e^{-f}
$$

Since both $u>0$ and $\varphi \geq 0$ are not identically zero, then combining (17) with (15), we have $\lambda_{1}(\Omega) \geq 0$ for all compact subdomains of $\Sigma$, then $\lambda_{1}(f, Q) \geq 0$, therefore $\Delta_{f}+q$ is stable.

We are now ready to give the proof of Theorem 1 .

Proof of Theorem 1 Since $\Phi: \Sigma^{n} \longrightarrow \mathbb{R}^{n+1}$ is a translating soliton, then the mean curvature $H$ satisfies $\Delta_{f} H+|A|^{2} H=0$ (see Proposition 3 in [1]). Since $H \geq 0$ and $\Sigma$ is a non-planar translating soliton, then $H$ is not identically zero, thus by Lemma 2, $\Sigma$ is stable and hence the weighted version of a result by Fischer-Colbrie and Schoen [2] guarantees there exists a non-constant positive $C^{2}$-function $\omega$ on $\Sigma$ such that

$$
\Delta_{f} \omega+|A|^{2} \omega=0 \text {. }
$$

As $\frac{H^{2}}{n} \leq|A|^{2}$ and $|A|$ satisfies (3), then

$$
\int_{B_{R}} H^{2} e^{-f} \leq n C R^{2}
$$


Then, by Corollary 1 and the condition that $H \geq 0$ and not identically zero, there is a constant $C_{1}>0$ such that

$$
H=C_{1} \omega \text {. }
$$

In particular $H>0$ everywhere on $\Sigma$. On the other hand, the Simons equation( see [1] or [6]) implies that

$$
|A|\left\{\Delta_{f}|A|+|A|^{2}|A|\right\}=|\nabla A|^{2}-\left.|\nabla| A\right|^{2} \geq 0 .
$$

Since $|A|$ satisfies (3), then by Lemma 1, $\exists C_{2} \geq 0$ such that

$$
|A|=C_{2} \omega \text {. }
$$

Besides that $\Sigma^{n}$ is not a hyperplane, then $|A|$ is not identically zero, thus $C_{2}>0$. Then by (20) and (22) we have $|A|^{2} H^{-2}=$ constant $>0$. In particular this function attains its local maximum on $\Sigma$. Theorem B in 6 says that $\Sigma$ is a grim hyperplane if and only if the function $|A|^{2} H^{-2}$ attains a local maximum. Therefore $\Sigma$ is a grim hyperplane.

We now prove Theorem 2 ,

Proof of Theorem 2, To prove Theorem 2, we will need a result of Huang-Wu[3]. Denote by $M_{+}$a connected component of $\{p \in M, H \geq$ 0 at $p\}$ that contains a point of positive mean curvature. We say that the

mean curvature $H$ changes signs through $\Gamma$ if $\Gamma$ is a connected component of $\partial M_{+}$and $\Gamma$ intersects the boundary of a connected component of $M \backslash \partial M_{+}$. Theorem 2 of Huang-Wu[3] $S \geq 0$, says that if $H$ changes sign along $\Gamma$ then $\Gamma$ is unbounded set. Since we have assumed that $H$ does not changes signs at infitiy, $H$ has a sign. Hence either

(1) $H \equiv 0$, or

(2) $H \geq 0$ but does not vanish at least one point.

In case (1), $\Sigma$ must be a hyperplane.

In case (2), if there is point $p \in \Sigma$, such that $S(p)=0$ then $|A|^{2}=$ $H^{2}-S \leq H^{2}$ and equality holds at $p$. Therefore the function $|A|^{2} H^{2}$ is well defined and attains its maximum at $p$. By Theorem B in [6] it must be a grim hyperplane.

\section{REFERENCES}

[1] Xu Cheng, Tito Mejia, and Detang Zhou, Simons-Type Equation for $f$-Minimal Hypersurfaces and Applications, J. Geom. Anal 25 (2015), no. 4, 2667-2686.

[2] Doris Fischer-Colbrie and Richard Schoen, The structure of complete stable surfaces in 3-manifolds of nonnegative scalar curvature, Comm. Pure Appl. Math. 33 (1980), no. 2, 199-211.

[3] Lan-Hsuan Huang and Damin Wu, Hypersurfaces with nonnegative scalar curvature, J. Differential Geom. 95 (2013), no. 2, 249-278. MR3128984

[4] Gerhard Huisken, Flow by mean curvature of convex surfaces into spheres, J. Differential Geom. 20 (1984), no. 1, 237-266. MR772132

[5] Peter Li and Jiaping Wang, Weighted Poincaré inequality and rigidity of complete manifolds, Ann. Sci. École Norm. Sup. (4) 39 (2006), no. 6, 921-982, DOI 10.1016/j.ansens.2006.11.001 (English, with English and French summaries). MR2316978 
[6] Francisco Martín, Andreas Savas-Halilaj, and Knut Smoczyk, On the topology of translating solitons of the mean curvature flow, Calc. Var. Partial Differential Equations 54 (2015), no. 3, 2853-2882, DOI 10.1007/s00526-015-0886-2. MR3412395

Instituto de Matemática e Estatística, Universidade Federal Fluminense, Niterói, RJ 24020, BrAziL

E-mail address: ditter.y.t@gmail.com

E-mail address: zhou@impa.br 